\title{
Cartografía de la vulnerabilidad de las aguas subterráneas a la contaminación por nitratos de fuentes difusas en la cuenca del río Ebro (N.E. de España)
}

\section{Mapping groundwater vulnerability to nitrate pollution from diffuse sources in the Ebro river basin (N.E. Spain)}

\author{
Mercedes Arauzo ${ }^{1, *}$, María Valladolid ${ }^{2}$, Gema García $^{1}$ \\ ${ }^{1}$ Instituto de Ciencias Agrarias, Consejo Superior de Investigaciones Científicas (CSIC), Serrano 115 dpdo. 28006 Madrid, \\ España. ORCID IDs: https://orcid.org/0000-0003-4113-8797, https://orcid.org/0000-0002-1715-3252 \\ ${ }^{2}$ Museo Nacional de Ciencias Naturales, Consejo Superior de Investigaciones Científicas (CSIC), José Gutiérrez Abascal 2, \\ 28006 Madrid, España. ORCID ID: https://orcid.org/0000-0002-7405-1105 \\ *Corresponding author: mercedes.arauzo@csic.es
}

\section{RESUMEN}

La contaminación por nitratos de fuentes difusas es una de las principales causas del deterioro de la calidad de los recursos hídricos subterráneos a escala global. Recientes investigaciones señalan la necesidad de minimizar la incertidumbre en la evaluación de la vulnerabilidad de las aguas subterráneas, mediante el desarrollo de metodologías robustas que permitan evaluar la vulnerabilidad bajo un enfoque fuente-vía-receptor en el contexto de la cuenca hidrológica. El procedimiento LU-IV (Arauzo, 2017) fue ideado para cartografiar la vulnerabilidad intrínseca de las aguas subterráneas (riesgos asociados al medio físico) y la vulnerabilidad específica a la contaminación por nitrato (riesgos asociados a los usos del suelo en territorios intrínsecamente vulnerables), con el objetivo final de mejorar la delimitación de las zonas vulnerables a la contaminación por nitrato (ZVN). Esta metodología utiliza parámetros sencillos y fácilmente disponibles que, combinados en un entorno de SIG, permite evaluar con precisión la vulnerabilidad (intrínseca y específica) en la totalidad de la cuenca de drenaje (área susceptible de drenar agua, potencialmente contaminada por nitrato, hacia un acuífero receptor).

La aplicación del procedimiento LU-IV a la cuenca hidrológica del río Ebro (N.E. de España) ha permitido determinar que una superficie de $18.757 \mathrm{~km}^{2}$ presenta un grado de vulnerabilidad específica a la contaminación por nitrato de alto a extremo, por lo que los territorios afectados podrían ser potencialmente designables como ZVN. Esta superficie difiere de los $9.796 \mathrm{~km}^{2}$ designados oficialmente como ZVN por las administraciones autonómicas con competencias para la designación de ZVN en la cuenca del Ebro. Si bien los territorios oficialmente designados coinciden en gran medida con los resultados de esta investigación, la aplicación del nuevo procedimiento ha permitido identificar un $50 \%$ más de territorios clasificables como zonas vulnerables. Las áreas aluviales presentaron el mayor grado de vulnerabilidad (tanto intrínseca como específica) afectando a la mayor parte de su territorio, lo que sugiere la necesidad de plantear una protección integral de todas las superficies aluviales, no sólo en la cuenca del Ebro, sino a escala nacional y europea.

Palabras clave: Procedimiento LU-IV; Sistemas de Información Geográfica (SIG); contaminación difusa; zonas vulnerables a la contaminación por nitrato (ZVN).

Recibido el 6 de febrero de 2020; Aceptado el 23 de abril de 2020; Publicado online el 25 de septiembre de 2020

Citation / Cómo citar este artículo: Arauzo, M. et al. (2020). Cartografía de la vulnerabilidad de las aguas subterráneas a la contaminación por nitratos de fuentes difusas en la cuenca del río Ebro (N.E. de España). Estudios Geológicos 76(2): e132. https://doi. org/10.3989/egeol.43868.586

Copyright: (C) 2020 CSIC. This is an open-access article distributed under the terms of the Creative Commons Attribution-Non Commercial (by-nc) Spain 4.0 License. 


\begin{abstract}
Nitrate pollution from diffuse sources is one of the main causes of groundwater quality deterioration on a global scale. Recent research suggests the need to minimize uncertainty in assessing groundwater vulnerability through the development of a robust methodology for the evaluation of vulnerability, using a source-pathway-receptor approach at basin scale. The LU-IV procedure (Arauzo, 2017) was devised for mapping intrinsic groundwater vulnerability (risks associated with the physical environment) and specific vulnerability to nitrate pollution (risks associated with land use in areas that are intrinsically vulnerable), with the final goal of improving the nitrate vulnerable zones (NVZ) delineation. This methodology uses simple and readily available parameters combined in a GIS environment and allows us to accurately assess groundwater vulnerability in the entire catchment area that drains water (potentially polluted by nitrate) into a receptor aquifer.

The application of the LU-IV procedure to the river Ebro basin (N.E. Spain) revealed a surface area of $18,757 \mathrm{~km}^{2}$ under high to extreme vulnerability to nitrate pollution, so that the affected territories could potentially be designated as NVZ. These results differ from the $9.796 \mathrm{~km}^{2}$ designated as NVZ by the Spanish regional administrations with competences in NVZ designations in the Ebro basin. Although the officially designated NVZ are consistent with the results of this research, the application of the new procedure allowed us to identify $50 \%$ more surface area that could be classified as NVZ. Alluvial areas showed the highest degree of vulnerability (both intrinsic and specific) affecting most of their territory, which suggests the need to propose an integral protection of all alluvial surfaces, not only of the Ebro basin, but also at national and European level.
\end{abstract}

Keywords: LU-IV procedure; Geographic Information Systems (GIS); diffuse pollution; nitrate vulnerable zones (NVZ).

\section{Introducción}

La contaminación por nitratos de fuentes difusas es una de las principales causas de la pérdida de calidad de los recursos hídricos en todo el planeta. En lo que se refiere a las aguas subterráneas, este tipo de contaminación se encuentra generalmente asociada a actividades agrícolas y ganaderas, con la dificultad de que, al desarrollarse de manera casi imperceptible, es difícil de detectar, combatir y prevenir.

En la Unión Europea (UE), la Directiva de Nitratos (91/676/EEC; Council of the European Communities, 1991) establece que las masas de aguas deben considerarse afectadas por contaminación de nitrato cuando su concentración excede los $50 \mathrm{mg} \mathrm{L}^{-1}$. La Directiva establece la obligatoriedad por parte de los Estados miembros de identificar las zonas cuya escorrentía o filtración afecte o pueda afectar a la contaminación por nitrato de las masas de agua. Estos territorios deben ser designados bajo la denominación de zonas vulnerables a la contaminación por nitrato (ZVN). En las ZVN deben implementarse programas de acción orientados a la recuperación de la calidad del agua y a la prevención de la contaminación, con emisión obligatoria de informes de control cuatrienales por parte de los Estados miembros. Sin embargo, en la UE todavía no se ha establecido un criterio común para la identificación y designación de ZVN, si bien se viene constatando la necesidad de realizar un esfuerzo adicional que permita obtener una mayor precisión en las designaciones, a fin de mejorar la efectividad de los programas de acción (Commission of the European Communities, 2007; Arqued, 2018).

Los mapas de vulnerabilidad de las aguas subterráneas basados en la aplicación de índices de vulnerabilidad mediante Sistemas de Información Geográfica (SIG) han sido ampliamente utilizados por las Administraciones durante las últimas décadas en la toma de decisiones sobre políticas de planificación de los recursos hídricos, si bien, todavía deben resolverse algunas lagunas metodológicas relacionadas con la inclusión o exclusión de determinados parámetros de los índices y las calificaciones y pesos relativos asignados a cada parámetro en la evaluación de riesgos (Kumar et al., 2015). Así, Machiwal et al. (2018) subrayan la necesidad de minimizar la incertidumbre en la evaluación de la vulnerabilidad mediante el desarrollo de una metodología robusta y global, que permita evaluar la vulnerabilidad de las aguas subterráneas en un entorno de SIG, bajo un enfoque fuente-vía-receptor (en el contexto de la cuenca hidrológica) y mediante procedimientos válidos para diferentes escalas espaciales, y condiciones hidrogeológicas y climáticas. En este contexto, el procedimiento LU-IV ha sido desarrollado (Arauzo, 2017) y optimizado (Arauzo et al., 2019) con el doble propósito de: (1) ofrecer una herramienta efectiva para cartografiar la vulnerabilidad intrínseca de las aguas subterráneas (que evalúa los riesgos asociados 
a factores del medio físico) y la vulnerabilidad específica a la contaminación por nitrato (que determina los riesgos de lixiviación de nitrato hacia las masas de agua subterránea asociados a los usos del suelo en territorios evaluados como intrínsecamente vulnerables) y (2) mejorar la identificación y designación de las ZVN.

El procedimiento LU-IV combina, mediante la aplicación de funciones lógicas de un SIG, un mapa de vulnerabilidad intrínseca (mapa IV, basado en el índice IV; Arauzo, 2017) y un mapa de los riesgos de pérdida de nitrato hacia las masas de agua subterránea asociados a los usos del suelo (mapa LU; Arauzo, 2017). Al comparar los resultados del procedimiento LU-IV con otros modelos paramétricos de vulnerabilidad, como DRASTIC (Aller et al., 1987) y GOD (Foster, 1987), mediante validaciones frente a las concentraciones medias de nitrato en puntos de control de las masas de agua subterráneas, el procedimiento LU-IV demostró una mayor eficacia en la evaluación de la vulnerabilidad intrínseca y específica, y una mejor capacidad predictiva de las zonas contaminadas por nitrato y de las zonas en riesgo (Arauzo et al., 2017). El método se distingue por las siguientes características diferenciales: (1) utiliza parámetros sencillos y fácilmente disponibles para alimentar el modelo, (2) evita la asignación de un peso específico para cada parámetro (al haberse verificado que el peso efectivo de los parámetros puede variar en función del tamaño y de las características de la cuenca o unidad territorial de análisis), (3) evalúa la totalidad de la cuenca hidrológica que puede potencialmente drenar agua hacia un acuífero receptor y (4) permite realizar actualizaciones del mapa de vulnerabilidad específica, a medida que se producen cambios en los usos del suelo y se generan versiones nuevas del mapa LU (Arauzo, 2017). Recientemente, el procedimiento LU-IV ha sido mejorado mediante el afino de los valores de riesgo que se asignan a los diferentes tipos de cultivo en el mapa LU (Arauzo et al., 2019). Estos nuevos valores "afinados" fueron obtenidos a partir de datos empíricos sobre los excedentes anuales de $\mathrm{N}$ por tipos de cultivo, procedentes de los informes anuales sobre el balance de $\mathrm{N}$ en la agricultura española (MAGRAMA, 2013, 2015a; MAPAMA, 2016, 2017). Al repetir las validaciones, utilizando las concentraciones medias de nitrato en puntos de control de las masas de agua subterránea, el procedimiento LU-IV mejorado (Arauzo et al., 2019) mostró nuevamente una mayor capacidad predictiva de las zonas contaminadas por nitrato y de las zonas en riesgo frente a otros modelos de vulnerabilidad, superando incluso los resultados de la primera versión del procedimiento LU-IV de 2017. El conjunto de estos resultados respalda el alto grado de fiabilidad del nuevo método.

En esta investigación hemos aplicado el procedimiento LU-IV (Arauzo, 2017), en su versión mejorada de Arauzo et al. (2019), para cartografiar y analizar la vulnerabilidad intrínseca y la vulnerabilidad específica a la contaminación por nitrato de las aguas subterráneas en la cuenca hidrológica del río Ebro (N.E. de España). A día de hoy, la tercera parte de las masas de agua subterránea de la Demarcación de la Cuenca del Ebro presenta áreas contaminadas por nitrato procedente de fuentes difusas (Confederación Hidrográfica del Ebro, 2019a, b), siendo los acuíferos aluviales los más gravemente afectados. Atendiendo a los requerimientos de la Directiva de Nitratos, las administraciones autonómicas de la cuenca del Ebro han realizado designaciones de ZVN durante las dos últimas décadas, sobre las que se vienen implementando programas de acción para la recuperación de la calidad del agua. Sin embargo, el actual marco normativo de la UE no explicita una metodología normalizada para la designación de $\mathrm{ZVN}$, por lo que la disparidad de criterios en las designaciones (European Commission, 2013) podría poner en riesgo la eficacia global de los programas de acción (Arauzo \& Valladolid, 2013; Worrall et al., 2009). Por ello, el objetivo principal de este trabajo consiste en abordar el análisis de la vulnerabilidad mediante una metodología contrastada y eficaz, como el procedimiento LU-IV, que integra los riesgos asociados a parámetros del medio físico y a los usos del suelo, y que cuenta con la característica diferencial de permitir evaluar la totalidad del territorio susceptible de drenar agua (contaminada o no) hacia un acuífero receptor, en el contexto de su cuenca hidrológica. Con ello pretendemos ofrecer a las Administraciones y los Agentes Sociales del sector agrario una herramienta que permita mejorar la precisión en la identificación de las ZVN y, en consecuencia, optimizar la implementación de los programas de acción para la recuperación de la calidad del agua, de acuerdo con la Directiva de Nitratos y con el Objetivo 6 de la 
Agenda 2030 para el Desarrollo Sostenible, de garantizar la disponibilidad y la gestión sostenible de los recursos hídricos (Economic and Social Council of the United Nations, 2018).

\section{Área de estudio y metodología}

\section{Área de estudio}

El área de estudio comprende el territorio de la cuenca hidrológica del río Ebro (N.E. de España; fig. 1). Presenta una extensión total de $85.660 \mathrm{~km}^{2}$, en la que se contabilizan 104 masas de agua subterránea principales que, en su conjunto, ocupan el $64 \%$ de la cuenca (fig. 1). De acuerdo a su litología, estas masas de agua corresponden mayoritariamente a acuíferos carbonatados, detríticos no aluviales y mixtos (59\% de la superficie de la cuenca) y a acuíferos aluviales (5\% de la superficie de la cuenca). El 35\% del conjunto de los acuíferos de la cuenca del Ebro presenta áreas contaminadas, con niveles de nitrato por encima de $50 \mathrm{mg} \mathrm{L}^{-1}$, si bien la contaminación por nitrato afecta al 95\% de los acuíferos aluviales (Confederación Hidrográfica del Ebro, 2019a, b). Las características específicas de las masas de agua subterránea pueden consultarse en MAGRAMA (2005).

La cuenca del Ebro limita al norte con los montes Vasco-Cantábricos y Pirineos, al sur con el Sistema Ibérico y al este con la cadena Costero-Catalana, dejando entre sí una zona deprimida que corresponde a la depresión del Ebro. Las áreas de montaña presentan un clima típicamente atlántico de montaña, con una precipitación media anual de 700 a $2000 \mathrm{~mm}$ (Botey et al., 2013), dependiendo de la zona y altitud. En el resto del territorio el clima varía desde el continental mediterráneo (con una clara gradación semiárida en el centro de la depresión del Ebro), donde la precipitación anual es de 300 a $400 \mathrm{~mm}$ (Botey et al., 2013), hasta el clima puramente mediterráneo del área del delta del Ebro, con precipitaciones anuales de 400 a $700 \mathrm{~mm}$ (Botey et al., 2013).

La contaminación difusa supone una de las principales presiones sobre la cuenca del Ebro. Entre los riesgos de contaminación difusa asociados a los usos

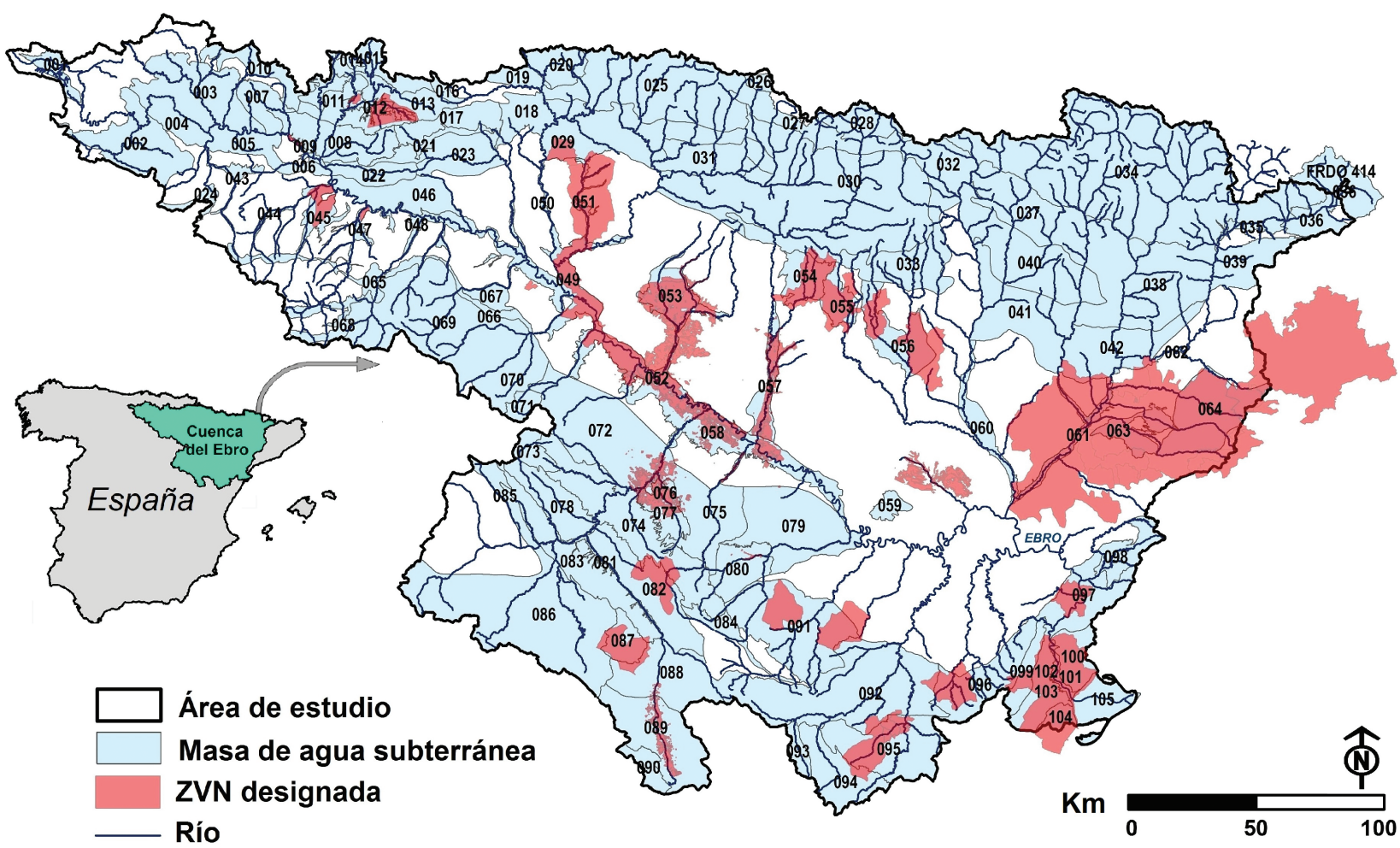

Figura 1.-Demarcación de la Cuenca de Ebro (N.E. de España). Las 106 masas de agua subterránea principales aparecen etiquetadas con los códigos numéricos asignados por la Confederación Hidrográfica del Ebro (2019b). También se representa las ZVN designadas oficialmente por las administraciones autonómicas de La Rioja, País Vasco, Navarra, Aragón y Cataluña. 
del suelo destacan las actividades agrícolas, siendo la fertilización la principal fuente exógena de compuestos nitrogenados (MAGRAMA, 2013, 2015a; MAPAMA, 2016, 2017). La cuenca del Ebro alberga una superficie agrícola de unos $30.000 \mathrm{~km}^{2}$, de los que $9.000 \mathrm{~km}^{2}$ son regadíos (SIOSE, 2011), que mayoritariamente se localizan en territorios aledaños a los cursos fluviales. Asimismo, cabe mencionar el problema derivado de la gestión de purines en la zona oriental de la cuenca (provincia de Lleida), con una alta densidad de cabaña ganadera, mayoritariamente de porcino.

Hasta hoy, cinco de las administraciones autonómicas con competencias para la designación de ZVN en la cuenca del Ebro (La Rioja, País Vasco, Navarra, Aragón y Cataluña) han designado como ZVN una superficie total de $9.796 \mathrm{~km}^{2}$ (Confederación
Hidrográfica del Ebro, 2019a, b), cuya distribución se muestra en la figura 1.

\section{Metodología}

La cartografía temática se elaboró utilizando las herramientas de Análisis Espacial de ArcGIS 10.3 for Desktop (ESRI, Redlands, CA, USA) y el sistema de referencia espacial ETRS89/UTM zona $30 \mathrm{~N}$. Para las capas en formato ráster se empleó un tamaño de pixel de $25 \mathrm{~m}$.

El procedimiento LU-IV (Arauzo, 2017), para cartografiar la vulnerabilidad intrínseca de las aguas subterráneas y la vulnerabilidad específica a la contaminación por nitrato, se desarrolla en dos pasos (fig. 2). Utiliza una escala única (de 1 a 10) para todos los parámetros, con cinco rangos de

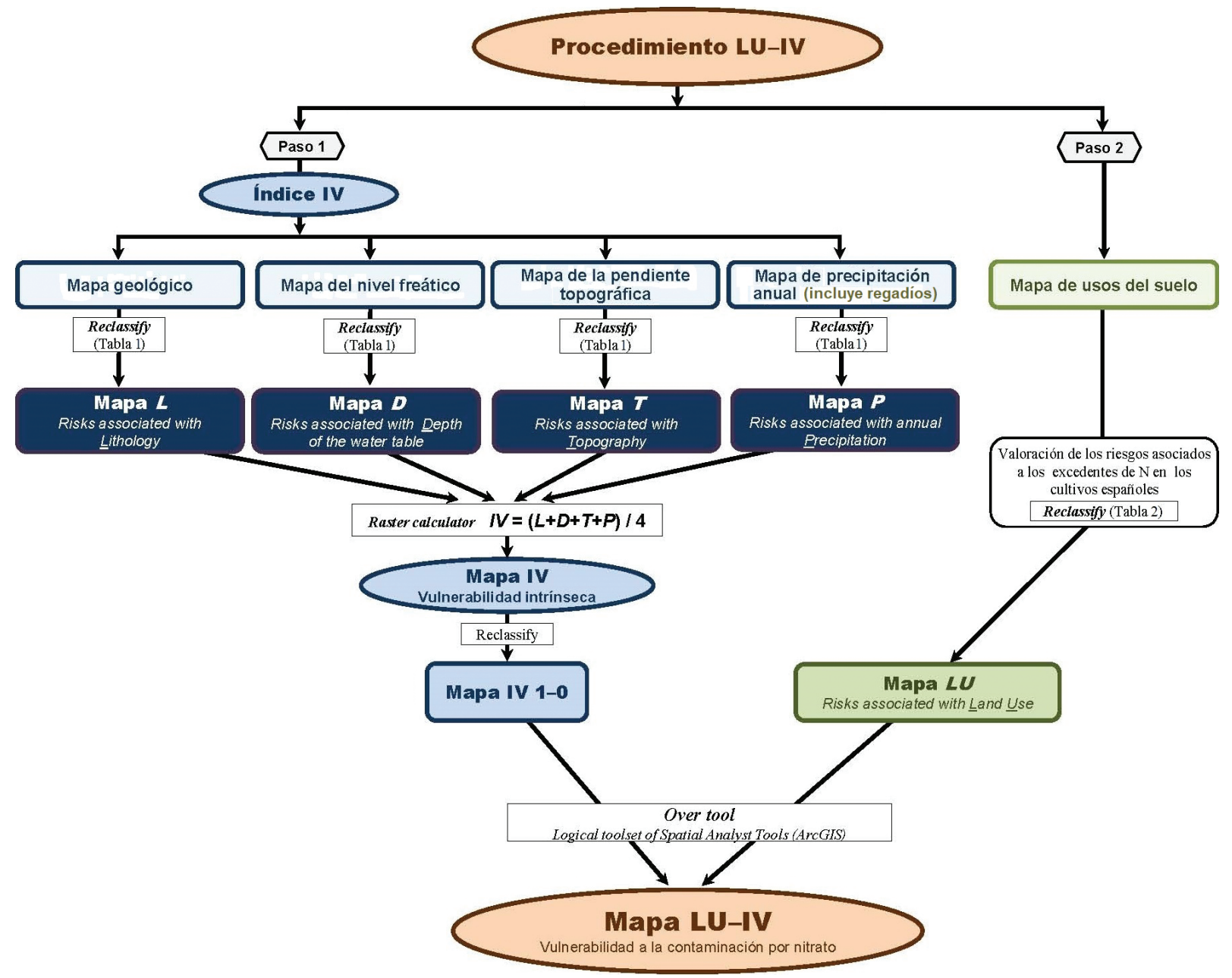

Figura 2.-Diagrama resumen del procedimiento LU-IV (extraído de la figura 3 en Arauzo et al., 2019). 
vulnerabilidad que corresponden a: riesgo inapreciable (valores 1-2), riesgo bajo (valores 3-4), riesgo medio o moderado (valores 5-6), riesgo alto (valores 7-8) riesgo extremo (valores 9-10) (tablas 1 y 2).

En el paso 1, el procedimiento genera el mapa ráster de vulnerabilidad intrínseca (mapa IV; fig. 2) a partir de cuatro parámetros del medio físico combinados mediante el siguiente algoritmo (índice IV):

$\mathrm{IV}=\frac{L+D+T+P}{4}$

Dónde, $L$ representa el riesgo asociado a la litología de la zona no saturada (Lithology of the vadose zone), $D$ representa el riesgo asociado a la profundidad del nivel freático (Depth to groundwater table),
$T$ representa el riesgo asociado a la pendiente topográfica (Topography) y $P$ representa el riesgo asociado a la precipitación media anual (Precipitation; que también incluye el riesgo por aportes extra de agua procedentes de los retornos de riego en las zonas de regadío). Para generar los mapas $L, D, T$ y $P$ de la cuenca del Ebro se siguió la metodología de Arauzo (2017), donde se describe la procedencia y justificación de los valores de referencia asignados a cada parámetro (tabla 1).

El mapa ráster $L$ se elaboró a partir de Mapa Geológico de España (Series MAGNA, Instituto Geológico y Minero de España) escala 1:50.000, en formato digital (Confederación Hidrográfica del Ebro, 2015), asignando los valores para $L$ de la tabla 1 .

Tabla 1.-Valores asignados a los rangos de los parámetros ambientales $L, D, P$ y $T$, que conforman el índice IV de vulnerabilidad intrínseca (extraídos de la tabla 3 en Arauzo et al., 2019).

\begin{tabular}{|c|c|c|c|}
\hline Litología de la zona no saturada " $L$ " & Valor & Profundidad del nivel freático “ $D$ ” (m) & Valor \\
\hline Calizas kársticas; caliches; gravas. & 10 & $\begin{array}{l}0-1 \text { (todas las profundidades para calizas kársticas, } \\
\text { caliches, gravas, calcarenitas y caliza de Creta) }\end{array}$ & 10 \\
\hline Calcarenitas; caliza de Creta. & 9 & $>1-3$ & 9 \\
\hline $\begin{array}{l}\text { Arenas aluviales y fluvio-glaciares; lavas volcánicas } \\
\text { recientes. }\end{array}$ & $7-8$ & $>3-5$ & 8 \\
\hline $\begin{array}{l}\text { Arenas eólicas; tobas volcánicas; formaciones ígneas/ } \\
\text { metamórficas fracturadas; formaciones volcánicas } \\
\text { antiguas; areniscas, conglomerados. }\end{array}$ & $5-6$ & $>5-10$ & 7 \\
\hline Limos aluviales, loess, till glacial; lutitas. & $3-4$ & $>10-13$ & 6 \\
\hline \multirow{5}{*}{$\begin{array}{l}\text { Arcillas; suelos residuales; formaciones ígneas/ } \\
\text { metamórficas no fracturadas y otros acuífugos. }\end{array}$} & $1-2$ & $>13-20$ & 5 \\
\hline & & $>20-33$ & 4 \\
\hline & & $>33-50$ & 3 \\
\hline & & $>50$ & 2 \\
\hline & & Ausencia de acuífero subyacente & 1 \\
\hline
\end{tabular}

\begin{tabular}{cccc}
\hline Precipitación media anual " $P$ " $(\mathbf{m m} \text { año })^{-1}$ & Valor & Pendiente topográfica “ $T^{\text {" }}(\%)$ & Valor \\
\hline$>900$ & 10 & $0-2$ & 10 \\
$>800-900$ & 9 & $>2-3$ & 9 \\
$>700-800$ & 8 & $>3-4$ & 7 \\
$>600-700$ & 7 & $>5-6$ & 6 \\
$>500-600$ & $6(7)^{\mathrm{a}}$ & $>6-9$ & 5 \\
$>400-500$ & $5(7)^{\mathrm{a}}$ & $>9-12$ & 4 \\
$>300-400$ & $4(7)^{\mathrm{a}}$ & $>12-15$ & 3 \\
$>200-300$ & $3(7)^{\mathrm{a}}$ & $>15-18$ & 2 \\
$>100-200$ & $2(7)^{\mathrm{a}}$ & $>18$ & 1 \\
$0-100$ & $1(7)^{\mathrm{a}}$ & $>$ & \\
\hline
\end{tabular}

${ }^{a}$ Los valores de los riesgos asociados al parámetro $P$ (precipitación) fueron ampliados en las zonas de regadío, al presentar éstas un riesgo adicional de aporte extra de agua procedente de los riegos; para ello, en las zonas de regadío con valores de precipitación entre 1 y 6 (riesgo entre inapreciable y moderado) el valor final asignado se elevó a un valor de 7 (riesgo alto). 
Tabla 2.-Valores de los riesgos asociados a los usos del suelo (parámetro LU; extraídos de la tabla 4 en Arauzo et al., 2019 ); los valores preferentes se muestran entre paréntesis.

\begin{tabular}{lr}
\hline Uso del Suelo $(\boldsymbol{L U})$ & \multicolumn{1}{c}{ Valor } \\
\hline Cultivo de hortícolas (hortalizas de hoja, flor y tallo; hortalizas de fruto, como tomate, guisante, judía verde, calabacín, & 10 \\
etc.; tubérculos, bulbos y raíces; cultivo de flores); cultivo de cítricos; cultivo de herbáceas forrajeras en regadío. & 9 \\
Cultivo de herbáceas forrajeras en secano; cultivo de frutales (no cítricos). & 8 \\
Cereales en regadío. & 7 \\
Cereales en secano. & $5-8$ (7) \\
Piscifactoría; granjas y explotaciones ganaderas; áreas urbanas. & 7 \\
Cultivo de almendros. & 6 \\
Viñedos; leguminosas (grano) en regadío; prados (regados). & 5 \\
Leguminosas (grano) en secano; prados y pastizales; cultivo de otras leñosas. & 4 \\
Olivar; cultivos industriales en regadío. & 3 \\
Cultivos industriales en secano. & $1-5$ (3) \\
Matorral. & $1-5$ (2) \\
Terreno no productivo. & 1 \\
Bosques y áreas naturales. & 4 \\
\hline
\end{tabular}

Para generar el mapa ráster $D$, se partió de capas vectoriales de puntos de las masas de agua (elaboradas a partir de la Red Piezométrica de la Confederación Hidrográfica del Ebro - CHE-), con los valores mínimos de profundidad del nivel freático en cada punto para el periodo 2005-2015 (información en formato Excel facilitada por la CHE). Los datos sobre profundidades de niveles freáticos fueron ampliados con información propia, hasta completar un total de 548 puntos de muestreo. Se usaron los valores mínimos de profundidad del freático al representar éstos el escenario menos favorable (de máxima vulnerabilidad) en el que la capa freática se encuentra más próxima a la superficie del terreno. Las interpolaciones a partir de las capas de puntos (para generar los rasters de profundad del freático) se hicieron acuífero a acuífero, asignando posteriormente (mediante Reclassify de Análisis Espacial de ArcGIS) los valores para $D$ de la tabla 1 .

El mapa ráster $T$ se creó a partir del mapa de la pendiente topográfica, generado a su vez a partir de un Modelo Digital del Terreno (MDT) de $25 \mathrm{~m}$ de resolución (IGN, 2018), al que se asignaron los valores para $T$ de la tabla 1 (mediante Reclassify).

El mapa ráster $P$ se elaboró (mediante interpolación) a partir de una capa vectorial de 490 puntos con los valores medios de precipitación anual para el periodo 1981-2010 (Botey et al., 2013), asignando posteriormente (mediante Reclassify) los valores correspondientes a los distintos rangos de $P$ (tabla 1). Dado que, además, en las zonas de regadío existe un riesgo adicional por los aportes extra de agua procedentes de los retornos de riego, el ráster $P$ de precipitación, se amplió incluyendo una valoración de los riesgos asociados a las superficies de regadío (identificadas a partir de SIOSE, 2011). En particular, nos fijamos en los cultivos de herbáceos en regadío (hortícolas, cereales, herbáceas forrajeras y otras herbáceas en régimen de regadío), generalmente regados mediante sistemas poco eficientes (aspersión o inundación; MAGRAMA, 2015b). Para ello, en las zonas de regadío coincidentes con valores de precipitación entre 1 y 6 , de riesgo inapreciable a moderado, el valor final asignado a cada pixel se elevaría a un valor de 7 , correspondiente a un riesgo alto (justificable por la sobrecarga efectiva que suponen los retornos de riego en la valoración del parámetro).

Para obtener el mapa de vulnerabilidad intrínseca (mapa IV), los rasters de los mapas $L, D, T$ y $P$ se combinaron aplicando el índice IV, mediante la herramienta Map Algebra de Análisis Espacial de ArcGIS.

En el paso 2, el procedimiento genera un ráster de vulnerabilidad específica a la contaminación por nitrato (mapa LU-IV; fig. 2) mediante la combinación del mapa de vulnerabilidad intrínseca (mapa IV, en su versión IV 1-0) y el mapa de riesgos asociados 
a los usos del suelo (mapa $L U$, que se explica más abajo). Para ello se utiliza la herramienta lógica Over de Análisis Espacial de ArcGIS.

Pero antes de aplicar Over, reclasificamos el mapa IV a valores 1 y 0 (mediante Reclassify). Para ello asignamos el valor 1 a los valores de IV entre 1 y 4 (riesgo inapreciable y bajo) y el valor 0 a los valores de IV entre 5 y 10 (riesgo medio, alto y extremo). Así obtuvimos el mapa ráster IV 1-0 (fig. 2), que permite diferenciar los territorios intrínsecamente vulnerables (con valor de pixel 0 ) de los no vulnerables (con valor de pixel 1).

Asimismo, para generar el mapa $L U$ (Land Use; mapa de riesgos asociados a los usos del suelo) se partió como mapa base del Mapa del Sistema de Información sobre Ocupación del Suelo de España SIOSE 2011, a escala 1:25.000 (SIOSE, 2011; IGN, 2015; de ahora en adelante referido como SIOSE). SIOSE divide el territorio con una malla continua de polígonos de cobertura única, cobertura compuesta, mosaico o asociación. Desde su primera versión, del año 2005, SIOSE ha sido actualizado en 2009 y 2011. Los shapefiles de SIOSE (2011) fueron recortados a la extensión del área de estudio y su tabla de atributos se completó con el parámetro $L U$ (tabla 2). En los polígonos de cobertura única (áreas homogéneas con un solo uso) se aplicó el mismo valor de calificación a todo el polígono. En los polígonos de cobertura compuesta, mosaico o asociación (áreas constituidas por una combinación de subpolígonos con diferentes usos) la calificación final asignada se calculó como la media aritmética ponderada de las calificaciones de uso en cada subpolígono (proporcionalmente a sus áreas respectivas). Los criterios de optimización para la asignación de los valores de $L U$ (tabla 2) se obtuvieron del estudio de Arauzo et al. (2019) y se encuentran fundamentados en el análisis de los datos sobre excedentes anuales de $\mathrm{N}$ en los diferentes tipos de cultivo, extraídos de los informes anuales sobre balances de $\mathrm{N}$ en la agricultura española para el periodo 2011-2015 (MAGRAMA, 2013, 2015a; MAPAMA, 2016, 2017). Del mapa $L U$ en formato vectorial se extrajo el mapa $L U$ en formato ráster.

Finalmente, para ejecutar la herramienta Over, el ráster IV 1-0 se asignó como la primera entrada de Over (fig. 2) y el ráster $L U$ como la segunda entrada (fig. 2). Over opera de manera que, para los valores de celda en la primera entrada (mapa IV 1-0) que son iguales a 1 (territorios intrínsecamente no vulnerables), el valor de salida será el mismo, es decir 1 (que corresponde a aquellos territorios en los que no es necesario aplicar restricciones de uso). Sin embargo, para los valores de celda que en la primera entrada son igual a 0 (territorios intrínsecamente vulnerables), la salida será la de la celda del ráster de la segunda entrada (mapa $L U$ ). Como resultado de la aplicación de esta herramienta lógica, obtuvimos el mapa de la vulnerabilidad específica a la contaminación por nitrato (mapa LU-IV), que completa el procedimiento LU-IV (fig. 2). A partir del mapa LU-IV, es posible identificar con precisión las ZVN.

Toda la información complementaria sobre el diseño, afino, validaciones y aplicabilidad del procedimiento LU-IV puede consultarse en Arauzo (2017) y Arauzo et al. (2019).

\section{Resultados y discusión}

\section{Vulnerabilidad intrínseca}

El mapa de vulnerabilidad intrínseca (índice IV; fig. 3) reveló que el $20 \%\left(16.959 \mathrm{~km}^{2}\right)$ de la superficie de la cuenca del Ebro presentaba niveles de riesgo de alto a extremo y el $37 \%\left(31.977 \mathrm{~km}^{2}\right)$ un nivel de riesgo medio (tabla 3 ), asociados a factores del medio físico (litología, hidrología, topografía y precipitación). En estas zonas, el agua de precipitación (y/o riego) es susceptible de percolar a través de la zona no saturada convirtiéndose en un vehículo potencial para el transporte vertical de los solutos (y para el transporte advectivo subsuperficial, en caso de existir pendiente) hasta las masas de agua subterránea.

Los mapas temáticos que conforman el mapa de vulnerabilidad intrínseca (fig. 3) revelan la escasa capacidad de atenuación del sustrato litológico (mapa $L$ ) y el alto nivel de exposición del manto freático (mapa $D$ ) en los acuíferos aluviales y en extensas superficies de los carbonatados. La mayor parte de los aluviales presentan, además, un alto riesgo asociado a su escasa pendiente topográfica (mapa $T$ ), dado que ocupan mayoritariamente las zonas más bajas y aplanadas de las cuencas, donde el agua tiende a acumularse. El riesgo asociado a la precipitación (mapa $P$ ) varía con la altitud y la zona climática. En el caso particular de los acuíferos 

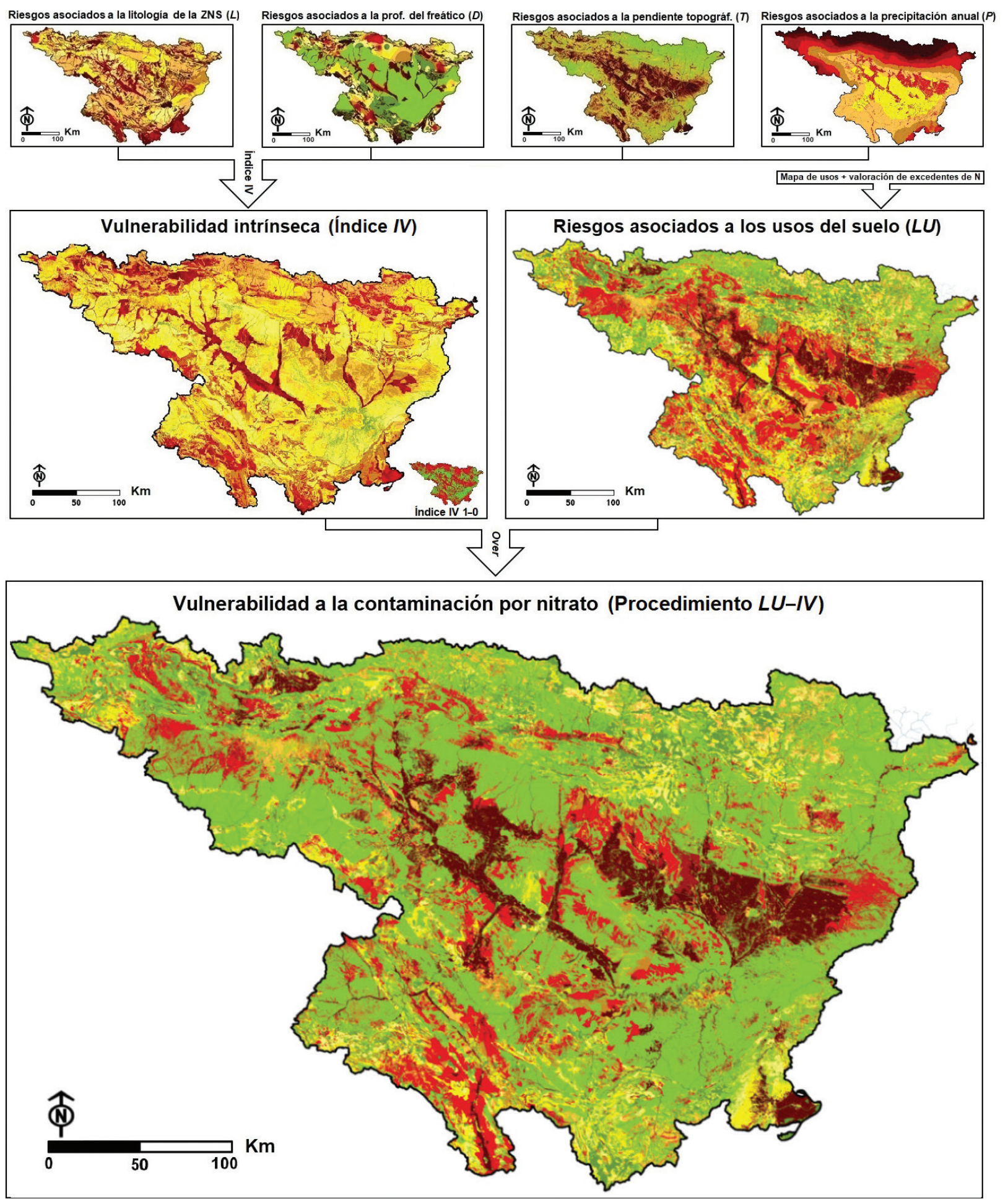

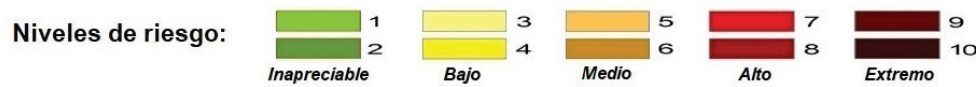

Figura 3.-Secuencia completa de los mapas temáticos que integran el procedimiento LU-IV. 
Tabla 3.-Zonas vulnerables en la cuenca del Ebro a partir de los mapas IV y LU-IV, comparadas con las ZVN designadas oficialmente por las administraciones autonómicas. Se muestran diferentes niveles de riesgo sobre la superficie total de la cuenca y sobre los acuíferos aluviales, acuíferos no aluviales y territorios sin masas de agua subyacentes (-zona seca-). El área potencialmente designable como ZVN de acuerdo al procedimiento LU-IV aparece subrayada.

\begin{tabular}{|c|c|c|c|c|}
\hline Mapa IV ( vulnerabilidad intrínseca) & $\begin{array}{l}\text { Área afectada } \\
\text { de la cuenca; } \\
\text { km }^{2}(\%)^{\mathrm{a}}\end{array}$ & $\begin{array}{c}\text { Afectación } \\
\text { sobre masas } \\
\text { aluviales; } \mathbf{k m}^{2}(\%)^{\mathbf{b}}\end{array}$ & $\begin{array}{c}\text { Afectación } \\
\text { sobre masas no } \\
\text { aluviales; } \mathbf{k m}^{2}(\%)^{\mathrm{c}}\end{array}$ & $\begin{array}{c}\text { Afectación } \\
\text { sobre zona seca; } \\
\text { km }^{2}(\%)^{\mathbf{d}}\end{array}$ \\
\hline Riesgo alto-extremo: & $16.959(20 \%)$ & $3.594(91 \%)$ & $13.216(26 \%)$ & $149(0,5 \%)$ \\
\hline Riesgo medio: & $31.977(37 \%)$ & $366(9 \%)$ & $21.028(42 \%)$ & $10.583(34 \%)$ \\
\hline \multicolumn{5}{|l|}{$\begin{array}{l}\text { Mapa LU-IV (vulnerabilidad específica a } \\
\text { la contaminación por nitrato) }\end{array}$} \\
\hline Riesgo alto-extremo (ZVN potenciales): & $18.757(22 \%)$ & $2.703(68 \%)$ & $8.818(18 \%)$ & $7.236(23 \%)$ \\
\hline Riesgo medio: & $6.131(7 \%)$ & $335(8 \%)$ & $4.371(9 \%)$ & $1.426(4 \%)$ \\
\hline \multicolumn{5}{|l|}{ ZVN designadas oficialmente } \\
\hline ZVN: & $9.796(11 \%)$ & $1.685(42 \%)$ & $4.625(9 \%)$ & $3.486(11 \%)$ \\
\hline
\end{tabular}

a Territorio afectado con relación a la superficie total de la cuenca del Ebro.

${ }^{\mathrm{b}}$ Territorio afectado con relación a la superficie total de las masas de agua subterránea aluviales.

${ }^{\mathrm{c}}$ Territorio afectado con relación a la superficie total de las masas de agua subterránea no aluviales.

${ }^{\mathrm{d}}$ Territorio afectado con relación a la superficie total del territorio sin acuíferos subyacentes.

aluviales de la depresión del Ebro, donde las precipitaciones son escasas (300-400 $\mathrm{mm}_{\text {año }}{ }^{-1}$; fig. 3), el riesgo asociado a los aportes por precipitación se incrementa notablemente cuando, además, consideramos los aportes extra de agua procedentes de la agricultura de regadío (susceptibles de generar retornos de riego con arrastre de solutos). El impacto potencial de los regadíos en las áreas aluviales es determinante, puesto que, de los aproximadamente $9.000 \mathrm{~km}^{2}$ de superficie de regadío de la cuenca del Ebro (SIOSE, 2011), el 30\% se distribuye sobre las superficies aluviales y el $70 \%$ restante sobre territorios aledaños a las mismas (con una distancia máxima de $8 \mathrm{~km}$ a los sistemas fluviales o a sus acuíferos aluviales asociados). Con respecto a los riesgos relacionados con la precipitación y la pendiente topográfica en los territorios sobre acuíferos carbonatados de áreas de montaña (Sistema Ibérico, Pirineos y montes Vasco-Cantábricos), se observaron niveles altos de riesgo asociados a las elevadas precipitaciones y niveles bajos de riesgo asociados a las pendientes pronunciadas (mapas $P$ y $T$; fig. 3 ), propias de los entornos montañosos.

De acuerdo con observaciones previas en espacios aluviales (Arauzo \& Valladolid, 2013; Arauzo et al., 2011), a partir del mapa de vulnerabilidad intrínseca (fig.3) se comprobó que el 91\% del área aluvial ( $3.594 \mathrm{~km}^{2}$ ) de la cuenca del Ebro presentaba niveles de riesgo de alto a extremo y el 9\% restante $\left(366 \mathrm{~km}^{2}\right)$ niveles de riesgo medio (tabla 3 ) asociados a factores del medio físico. La elevada vulnerabilidad intrínseca en la totalidad de la extensión aluvial, sugiere la necesidad de implementar mecanismos de protección en todos los territorios aluviales. Por otra parte, el análisis de la vulnerabilidad intrínseca en los territorios sobre las masas de agua no aluviales (acuíferos carbonatados, detríticos y mixtos) reveló niveles de riesgo de alto a extremo en el $26 \%$ de su superficie $\left(13.216 \mathrm{~km}^{2}\right)$ y niveles de riesgo medio en el $42 \%\left(21.028 \mathrm{~km}^{2}\right)$ (tabla 3$)$. $\mathrm{Si}$ bien, en este caso, la superficie relativa afectada resulta menor que la de los territorios aluviales, su superficie efectiva es muy superior, por lo que incluso los territorios en riesgo medio deben ser tenidos en cuenta en la etapa final del paso $1 \mathrm{del}$ procedimiento LU-IV (generación del mapa IV 1-0; fig. 2 y 3). Por último, el análisis de la vulnerabilidad intrínseca en los territorios sin masas de agua subterránea subyacentes (tabla 3) mostró niveles de riesgo de alto a extremo en el $0,5 \%\left(149 \mathrm{~km}^{2}\right)$ de su superficie, mientras que en el $34 \%\left(10.583 \mathrm{~km}^{2}\right)$ se alcanzaron niveles de riesgo medio. Teniendo en cuenta estas consideraciones, para representar los territorios intrínsecamente vulnerables a través del mapa IV 1-0 (fig. 2 y 3 ) se adoptó un criterio conservador, incluyendo en la elaboración del mapa, tanto los territorios con niveles de riesgo alto y extremo (valores del índice IV de 7 a 10), como los de riesgo 
medio (valores 5 y 6 ). Con esta medida se garantizó que no quedasen excluidas del procedimiento de análisis aquellas zonas que, aun presentando niveles medios de vulnerabilidad intrínseca (paso 1 del procedimiento), pudiesen encontrarse expuestas a usos del suelo de alto riesgo (paso 2 del procedimiento).

\section{Vulnerabilidad específica}

El mapa de vulnerabilidad específica a la contaminación por nitrato (fig. 3) permitió determinar que el $22 \%\left(18.757 \mathrm{~km}^{2}\right)$ de la superficie de la cuenca del Ebro presentaba niveles de riesgo de alto a extremo y el $7 \%\left(6.131 \mathrm{~km}^{2}\right)$ niveles de riesgo medio (tabla $3)$. Del conjunto del territorio afectado, el $68 \%$ de las áreas aluviales $\left(2.703 \mathrm{~km}^{2}\right)$ presentó niveles de riesgo de alto a extremo y el $8 \%\left(335 \mathrm{~km}^{2}\right)$ niveles medios (tabla 3), constatándose que los territorios aluviales son los más vulnerables (en superficie relativa) a la contaminación por nitrato. En general, sólo las zonas aluviales de las áreas de las cabeceras de cuenca, protegidas por bosques o áreas naturales, se ven libres de riesgos significativos. Estos datos, sumados al alto grado de vulnerabilidad intrínseca en el 100\% de los territorios aluviales, sugieren la necesidad de plantear una protección integral de la totalidad de los mismos, no sólo en la cuenca del Ebro, sino a escala nacional y europea. Por otra parte, el $18 \%$ de los territorios sobre masas de agua no aluviales $\left(8.818 \mathrm{~km}^{2}\right)$ presentó niveles de riesgo de alto a extremo y el $9 \%\left(4.371 \mathrm{~km}^{2}\right)$ niveles medios. Por último, en el $23 \%$ de los territorios sin masas de agua subterránea subyacente $\left(7.236 \mathrm{~km}^{2}\right)$ se dieron niveles de riesgo de alto a extremo y en el $4 \%\left(1.486 \mathrm{~km}^{2}\right)$ niveles de riesgo medio (tabla 3 ). La información sobre zonas de vulnerabilidad específica elevada en territorios de la cuenca en los que no existen masas de agua subterránea subyacente, pero desde los que cabe la posibilidad de que el agua de drenaje se desplace por escorrentía subsuperficial hasta acuíferos receptores de cotas más bajas, constituye una aportación novedosa del procedimiento LU-IV que hasta ahora nunca había sido contemplada por otros índices de vulnerabilidad (Arauzo, 2017). En el caso que nos ocupa, estas zonas representan el $8,4 \%$ del territorio de la cuenca del Ebro.

Si analizamos los pros y contras del uso del SIOSE como mapa base para generar el mapa $L U$ del procedimiento LU-IV (paso 2), cabría referir que la mayor limitación es que no permite distinguir entre las coberturas de diferentes tipos de cultivos herbáceos, agrupándolos a todos bajo dos únicos códigos descriptores. Afortunadamente, es posible distinguir entre herbáceos en regadío y herbáceos en secano mediante los descriptores de atributos, lo que compensa en cierta medida esa limitación. Por ello, como solución de compromiso, para la generación del mapa LU se aplicó un valor 9 (riesgo extremo) a los cultivos herbáceos en regadío (mayoritariamente hortícolas, pero también forrajeras y cereales en regadío; tabla 2) y un valor 7 (riesgo alto) a los herbáceos de secano (mayoritariamente cereales en secano; tabla 2). Esta solución conduce a sobrevalorar el riesgo de otras herbáceas, como las leguminosas en grano (que serían valoradas como de riesgo alto, en vez de riesgo medio; tabla 2) y los cultivos industriales (que aparecerían como de riesgo alto, en vez de riesgo bajo; tabla 2). Estos dos grupos de cultivos representan, respectivamente, el $4 \%$ y $11 \%$ de la superficie total de cultivos herbáceos (MAGRAMA, 2015b), por lo que el desajuste puede considerarse asumible, dadas las otras muchas ventajas que ofrece el SIOSE. Como contrapartida, el SIOSE proporciona información detallada sobre coberturas artificiales, lo que permite una valoración exhaustiva de territorios con granjas, explotaciones ganaderas, piscifactorías y áreas urbanas. Otro aspecto interesante del SIOSE tiene que ver con su escala, de 1:25.000, que ofrece la posibilidad de trabajar con una exactitud posicional de $2,5 \mathrm{~m}$. Por otra parte, sus actualizaciones periódicas (hasta ahora existen versiones de 2005, 2009 y 2011), permitirán hacer reajustes del mapa de vulnerabilidad específica y de las ZVN, a medida que se produzcan modificaciones en los usos de alto riesgo en el mapa de usos del suelo, lo que puede ser de particular interés en la elaboración de los informes cuatrienales sobre el estado y evolución de las ZVN. Como alternativa al SIOSE, la versión digital del Mapa de Cultivos y Aprovechamientos de España 2000-09 (MARM, 2009) obtuvo resultados similares, en cuanto a eficacia y fiabilidad, como mapa base para elaborar el mapa $L U$ (Arauzo et al., 2019).

Respecto a la asignación de valores para el parámetro $L U$ (riesgos asociados a los usos del suelo; tabla 2), que se fundamentó en el análisis de los 
excedentes anuales de $\mathrm{N}$ en los diferentes tipos de cultivo a escala nacional, para el periodo 2011-2015 (Arauzo et al., 2019; datos extraídos de los informes anuales sobre balances de $\mathrm{N}$ en la agricultura española de MAGRAMA, 2013, 2015a, y MAPAMA, 2016, 2017), cabe referir que los valores asignados podrían sufrir ciertos reajustes si se contemplan otros periodos, o se utilizan datos específicos para diferentes áreas del país, debiendo tenerse en cuenta su naturaleza variable y dependiente de las dosis anuales de fertilización nitrogenada. No obstante, los tipos de cultivo valorados como de riesgo alto o extremo (tabla 2), lo suelen ser para la mayoría de las zonas y periodos, tal como se constata en los sucesivos informes anuales sobre balances de $\mathrm{N}$ en la agricultura española.

\section{Zonas Vulnerables a la contaminación por nitrato}

A partir del mapa de vulnerabilidad específica a la contaminación por nitrato obtenido mediante procedimiento LU-IV (fig. 3), fue posible determinar que una superficie de $18.757 \mathrm{~km}^{2}$ podría ser potencialmente designable como ZVN (tabla 3). El territorio afectado corresponde al $22 \%$ de la superficie de la cuenca hidrológica del Ebro. Esta superficie es prácticamente el doble que la superficie de $9.796 \mathrm{~km}^{2}$ de ZVN designadas oficialmente por las administraciones autonómicas con competencias de designación en la cuenca del Ebro (11\% de la superficie de la cuenca; fig. 1; tabla 3). Si bien los territorios oficialmente designados como ZVN coinciden en gran medida con los resultados de esta investigación (fig. 4), la aplicación del procedimiento LU-IV ha permitido identificar zonas vulnerables que todavía no habían sido desveladas hasta ahora. Asimismo, ha evidenciado algunos territorios designados en los que se podría rebajar el nivel de exigencia. Al analizar el grado de coincidencia entre los rasters de ZVN potenciales (resultado del procedimiento LU-IV) y de ZVN oficiales (designadas por las administraciones autonómicas) en la cuenca del Ebro (fig. 4), se encontró que el $79 \%$ del territorio presentaba valoraciones coincidentes en ambas aproximaciones,

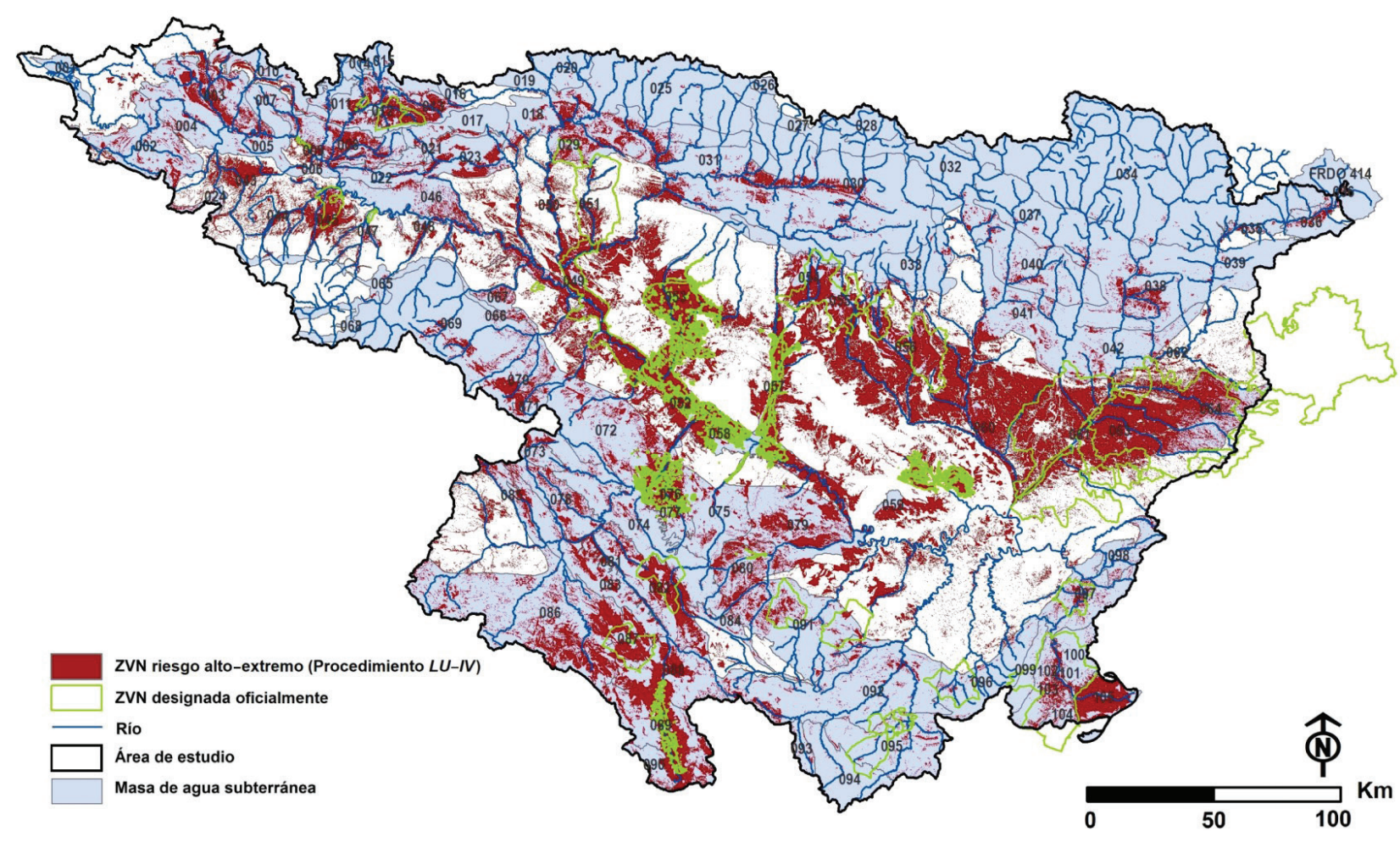

Figura 4.-ZVN potenciales (en rojo), estimadas mediante el procedimiento LU-IV. También se muestran las ZVN designadas oficialmente por las administraciones autonómicas (contornos en verde). 
correspondiendo el $73 \%$ a zonas valoradas como no vulnerables y el $6 \%$ a zonas consideradas ZVN. Por otra parte, un 5\% del territorio designado como ZVN oficiales no mostró correspondencia con el ráster de ZVN potenciales. Mientras que el $16 \%$ del territorio identificado como $\mathrm{ZVN}$ potenciales, no presentó correspondencia con el ráster de ZVN oficiales.

\section{Conclusiones}

La aplicación del procedimiento LU-IV a la cuenca hidrológica del río Ebro ha permitido determinar que una superficie de $18.757 \mathrm{~km}^{2}(22 \%$ de la superficie de la cuenca) presenta un grado de vulnerabilidad específica a la contaminación por nitrato de alto a extremo, por lo que los territorios afectados podrían ser potencialmente designables como ZVN. Esta superficie es superior a la superficie de $9.796 \mathrm{~km}^{2}$ de ZVN designadas oficialmente por las administraciones autonómicas de la cuenca del Ebro (11\% de la superficie de la cuenca). Si bien los territorios oficialmente designados como ZVN coinciden en gran medida con los resultados de esta investigación, la aplicación del nuevo procedimiento LU-IV ha permitido identificar un $50 \%$ más de territorios clasificables como zonas vulnerables, que todavía no habían sido desvelados. Los territorios aluviales presentaron el mayor grado de afectación, tanto por su vulnerabilidad intrínseca como por su vulnerabilidad específica, lo que sugiere la necesidad de plantear una protección integral de la totalidad de los mismos, no sólo en la cuenca del Ebro, sino a escala nacional y europea.

El desarrollo de esta investigación ha constituido un primer paso imprescindible en el análisis espacial de la vulnerabilidad específica a la contaminación por nitrato en la cuenca del río Ebro. Los resultados obtenidos han permitido mejorar el conocimiento y delimitación de las ZVN, y pueden contribuir a maximizar la eficacia de los programas para la recuperación de las masas de agua subterránea contaminadas por nitrato, de acuerdo a los requerimientos establecidos en la Directiva de Nitratos.

\section{AGRADECIMIENTOS}

Agradecemos a la Agencia Estatal de Investigación del Ministerio Español de Ciencia, Innovación y Universidades y al Fondo Europeo de Desarrollo Regional la financiación del Proyecto CGL2016-81110-R (AEI/FEDER, UE) en el que se enmarca esta investigación. Las capas vectoriales de Masas de Agua Subterránea, Red Fluvial, Cuenca del Ebro, Puntos Piezométricos, Mapa Geológico y Zonas Vulnerables de la cuenca del Ebro, proceden del Geoportal SITEbro de la Confederación Hidrográfica del Ebro (CHE). Asimismo, las capas vectoriales del Sistema de Información sobre Ocupación del Suelo de España SIOSE 2011 y el MDT de 25 m se obtuvieron del Centro de Descargas del Instituto Geográfico Nacional (IGN). La mayor parte de los datos sobre niveles freáticos nos los proporcionó la CHE en formato Excel. Por todo ello, nuestro agradecimiento a la CHE y el IGN, sin cuyas contribuciones no hubiese sido posible el desarrollo de este trabajo de investigación.

\section{Referencias}

Aller, L.; Bennet, T.; Lehr, J.H. \& Petty, R.J. (1987). DRASTIC. A standardized system for evaluating groundwater pollution potential using hydrogeologic settings. U.S. Environmental Protection Agency EPA/600/2-87-035, Oklahoma, 622 pp.

Arauzo, M.; Valladolid. M. \& Martínez-Bastida, J.J. (2011). Spatio-temporal dynamics of nitrogen in river-alluvial aquifer systems affected by diffuse pollution from agricultural sources: implications for the implementation of the Nitrate Directive. Journal of Hydrology, 411: 155-168. https://doi.org/10.1016/j. jhydrol.2011.10.004

Arauzo, M. \& Valladolid, M. (2013). Drainage and $\mathrm{N}$-leaching in alluvial soils under agricultural land uses: Implications for the implementation of the EU Nitrates Directive. Agriculture, Ecosystems \& Environment, 179: 94-107. https://doi.org/10.1016/j. agee.2013.07.013

Arauzo, M. (2017). Vulnerability of groundwater resources to nitrate pollution: a simple and effective procedure for delimiting Nitrate Vulnerable Zones. Science of the Total Environment, 575: 799-812. https://doi.org/10.1016/j.scitotenv.2016.09.139

Arauzo, M.; García, G. \& Valladolid, M. (2019). Assessment of the risks of N-loss to groundwater from data on N-balance surplus in Spanish crops: An empirical basis to identify Nitrate Vulnerable Zones. Science of the Total Environment, 696: 133713. https://doi. org $/ 10.1016 / j$. scitotenv. 2019.133713

Arqued, V.M. (2018). Es probable que se amplíen las zonas declaradas como vulnerables por la contaminación con nitratos agrícolas. Tierras, 268: 120-122.

Botey, R.; Guijarro, J.A. \& Jiménez, A. (2013). Valores normales de precipitación mensual 1981-2010. 55 págs. Dirección de Producción e Infraestructuras, Agencia Estatal de Meteorología (AEMET), Ministerio de Agricultura, Alimentación y Medio Ambiente, Madrid, $55 \mathrm{pp}$.

Commission of the European Communities (2007). Report from the Commission to the Council and the 
European Parliament on implementation of Council Directive 91/676/EEC concerning the protection of waters against pollution caused by nitrates from agricultural sources for the period 2000-2003 \{SEC (2007) 339\}./* COM/2007/0120 final*/, Brussels.

Confederación Hidrográfica del Ebro (2015). Mapa Geológico de España [mapas en formato digital] 1:50.000. Series MAGNA, Instituto Geológico y Minero de España. Madrid. http://iber.chebro.es/geoportal/ (acceso el 29 de abril de 2015).

Confederación Hidrográfica del Ebro (2019a). Aguas afectadas por nitratos de origen agrario (2012-2015) http:/www.chebro.es/contenido.visualizar.do?idC ontenido=19441\&idMenu=3811/ (acceso el 2 de diciembre 2019).

Confederación Hidrográfica del Ebro (2019b). Inicio SITEbro. Geodatos. Descarga de datos cartográficos en formato de fichero agrupados por temáticas; Masas de Agua Subterránea; Red Fluvial; Puntos Piezométricos; Mapa Geológico; Zonas Vulnerables. http://iber.chebro.es/geoportal/index.htm (acceso el 2 de diciembre 2019).

Council of the European Communities (1991). Directive 91/676/EEC concerning the protection of waters against pollution caused by nitrates from agricultural sources. 12 December 1991. Official Journal of the European Union L 375, 31/12/1991, Brussels.

Economic and Social Council of the United Nations (2018). Special edition: progress towards the Sustainable Development Goals. Report of the Secretary-General. Chapter II: Where we are in the achievement of the Sustainable Development. Goal 6, Ensure availability and sustainable management of water and sanitation for all. https://undocs.org/E/2019/68 (acceso el 29 julio de 2019).

European Commission (2013). Report from the Commission to the Council and the European parliament on implementation of Council Directive 91/676/EEC concerning the protection of waters against pollution caused by nitrates from agricultural sources based on Member State reports for the period 2008-2011. 13 pp. Commission Staff Working Document, Brussels.

Foster, S.S.D. (1987). Fundamental concepts in aquifer vulnerability, pollution risk and protection strategy. In: Vulnerability of Soil and Groundwater to Pollution (Duijvanbooden, W. Van \& Waegeningh, H.G. Van, Eds.), Proceedings and Information No. 38, TNO Committee on Hydrological Research, the Netherlands, 69-86.

IGN (2015). Sistema de Información de Ocupación del Suelo en España. Documento Técnico SIOSE 2011. Versión 1.1. Instituto Geográfico Nacional, Madrid.

IGN (2018). Modelo Digital del Terreno - MDT25 [mapas en formato ráster de $25 \mathrm{~m}$ de resolución]. Instituto Geográfico Nacional, Madrid. http://centrodedescargas.cnig.es/CentroDescargas/index.jsp (acceso el 20 de abril de 2018).
Kumar, P.; Bansod, B.K.S.; Debnath, S.K.; Kumar T., P. \& Ghanshyam, C. (2015). Index-based groundwater vulnerability mapping models using hydrogeological settings: A critical evaluation. Environmental Impact Assessment Review, 51: 38-49. https://doi. org/10.1016/j.eiar.2015.02.001

Machiwal, D.; Jha, M.K.; Singh, V.P. \& Mohan, C. (2018). Assessment and mapping of groundwater vulnerability to pollution: Current status and challenges. Earth-Science Reviews, 185: 901-927. https://doi. org/10.1016/j.earscirev.2018.08.009

MAGRAMA (2005). Caracterización de las masas de agua subterránea de las cuencas intercomunitarias. Tomo V. Ministerio de Agricultura, Alimentación y Medio Ambiente, Madrid.

MAGRAMA (2013). Balance del nitrógeno en la agricultura española, año 2011. Ministerio de Agricultura y Pesca, Alimentación y Medio Ambiente, Secretaría General Agricultura y Alimentación, Dirección General de Producciones y Mercados Agrarios, Madrid.

MAGRAMA (2015a). Balance del nitrógeno en la agricultura española, año 2013. Ministerio de Agricultura y Pesca, Alimentación y Medio Ambiente, Secretaría General Agricultura y Alimentación, Dirección General de Producciones y Mercados Agrarios, Madrid.

MAGRAMA (2015b). Informe sobre regadíos en España. Ministerio de Agricultura y Pesca, Alimentación y Medio Ambiente, Secretaría General Técnica, Subdirección General de Estadística, Madrid.

MAPAMA (2016). Balance del nitrógeno en la agricultura española, año 2014. Ministerio de Agricultura y Pesca, Alimentación y Medio Ambiente, Secretaría General Agricultura y Alimentación, Dirección General de Producciones y Mercados Agrarios, Madrid.

MAPAMA (2017). Balance del nitrógeno en la agricultura española, año 2015. Ministerio de Agricultura y Pesca, Alimentación y Medio Ambiente, Secretaría General Agricultura y Alimentación, Dirección General de Producciones y Mercados Agrarios, Madrid.

MARM (2009). Mapa de Cultivos y Aprovechamientos de España 2000-09 [mapas en formato digital] 1:50.000. Ministerio de Medio Ambiente, Medio Rural y Marino de España, Madrid.

SIOSE (2011). SIOSE 2011 Sistema de Información sobre Ocupación del Suelo de España [mapas en formato digital] 1:25.000. CInstituto Geográfico Nacional, Madrid. http://centrodedescargas.cnig.es/CentroDescargas/busquedaSerie.do? codSerie=SIOSE (acceso el 13 de octubre de 2018).

Worrall, F.; Spencer, E. \& Burt, T.P. (2009). The effectiveness of nitrate vulnerable zones form limiting surface water nitrate concentrations. Journal of Hydrology, 370, 21-28. https://doi.org/10.1016/j. jhydrol.2009.02.036 